\title{
Health Informatics Sciences Lessons from the Past 50 Years and Future Prospects
}

\author{
Francis Roger France* \\ University of Louvain Medical School, Europe \\ Received: March 20, 2018; Published: April 05, 2018 \\ *Corresponding author: Francis Roger France, University of Louvain Medical School, Europe, Email: fhrf@rogerfrance.com
}

\section{Opinion}

\section{(1967 - 2017) Advances in Health Sciences and Informatics Technologies}

During the last fifty years, medicine made great progresses. Research and technologies contributed to an extraordinary development of knowledge and concepts. It might have been the best period in history for physicians to practice: laboratory tests, biochemistry, genomics, digitized imaging, public health indicators and other developments induced confidence of patients by establishing well documented diagnoses. Treatments became more efficient, with novelties, like heart transplant, chemotherapy and immuno-therapy. During this period, social security systems allowed a wider access of populations to health care. Patients were more confident in medicine and physicians felt free. Simultaneously, computer technology and information sciences developed rapidly. Data input through punched cards was replaced by PCs interconnected through networks to large computers. New softwares, data mining and knowledge bases changed the world. The World Wide Web, Internet, artificial intelligence and robotics became accessible. New hospitals using these modern technologies were planned in industrialized countries.

In 1967, the University of Louvain decided to build a teaching hospital (Saint-Luc) in Brussels (Belgium), where an eHealth unit record should be created. I was asked to be educated, therefore, in general internal medicine in order to have a holistic approach of patients, as well as in health computer sciences, biometry and epidemiology, in order to make population studies, at the University of Minnesota School of Public Health and at the Mayo Clinic. A pilot project introduced standards in Belgium for a "medical record summary", unique by patient with population based objectives. International classifications (ICD-9-CM) and nomenclatures (SNOMED) were recommended for diagnoses and interventions, in order to study hospital activities, performance and quality of care, mortality and morbidity trends. This model lead to the European Minimum Basic Data Set (MBDS, 1982) [1], enabling to link diagnoses to hospital activities among EEC member countries and allowing new financing systems based on case-mix such as Diagnosis Related Groups (DRGs) [2].

\section{Impact of Informatics on Clinical Practice}

Most technologies that were repetitive could be computerized, like laboratory tests, ECGs, EEGs, EMGs. Digital imaging improved the vision of lesions. Computerized Aid to medical diagnosis, like the work of Ted Shortliffe for infectious diseases and cancers or diagnostic scores proposed by F.T.de Dombal for abdominal pathologies were fascinating but remained punctual applications. Lawrence Weed [3] had a larger impact on current implicit clinical practice by promoting the "Problem oriented medical record", a structured information model to build electronic records that could help physicians to make explicit their diagnostic reasoning.

\section{Lessons Learned}

First lesson learned: A structured Problem oriented electronic medical record was developed in St-Luc hospital, with a Summary divided in 4 parts:

a) Patient's complaints and symptoms

b) Inactive problems (like appendectomy)

c) Hypotheses of diagnoses and unsolved problems

d) Active pathologies and problems. Applying this method systematically proved to be very useful for diagnoses and treatments. The quality of medical reports improved and was highly appreciated.

However, this holistic approach requires a strict discipline, a good knowledge of medicine and a human interaction with the patient. This proved difficult to generalize in the practice of most physicians. They were not educated to this methodology. During the last fifty years, there were more and more "Hyper-specialists" and less and less general internists, at the despair of many patients. If the clinical summary does not reflect a global view of patient problems, computerized results might be biased. The quality 
of patients information processing depends on the precision, exactitude and comprehensiveness of the data base. For example, obesity would often not be noted as risk factor in the MBDS if patients were hospitalized for heart infarction. Maybe that we are still in a too early stage of development of the eHealth record, The actual digitalization of most health data by mHealth apps (mobile portable mini-computers measuring pulse, blood pressure,...) might improve eHealth record input, but for complex health problems, a human judgment will always be needed.

Second Lesson Learned: The application of the MBDS to hospital financing was much more appreciated by economists and operation researchers than by clinicians, poorly motivated to measure public health objectives such as clinical epidemiology or performance and quality of care. They used it, however, for clinical research. International comparisons of patient data sets were difficult to obtain, mainly for the description of procedures, given national variations in coding. Procedures are linked to payment systems. A very detailed billing data input in a Social Security system, like in Belgium, cannot find an equivalent description in National Health Services, like in United Kingdom.

\section{New Trends and Future Prospects}

eHealth (Electronic Health): Refers to all health fields, but identifies in Belgium all health data available by citizen (hospital records summaries, general practitioners data, laboratory, imaging and drugs prescribed information). It is managed by Public Health Federal authorities and can only be accessed after authorization checking. It might help to avoid tests overuse but could underestimate who has really access to what. Strict personal health data protection rules should be checked.
Telemedicine (Diagnosis, Treatment and Follow-up at Distance): Could save lives (by detecting, for example, atrial transient fibrillation) and induced already a return of GP's in rural areas. Laws have to be adapted in order to distinguish professional liability (physician at distance, on site, and digitized tools).

Industrial Mega- Information Systems: (both administrative and patient care oriented packages with multiple interrelated applications, such as scheduling appointments, standardized procedures for care, billing,...) might rationalize health care processes but seem to prioritize financial profit rather than quality of care.

Third Lesson Learned: Such systems might have serious adverse effects on health professions and their freedom of practice. Physicians resist less and less to stricter schedules for care and work overload, as documented by the rising number of "burn-out" cases and defections of medical practice. Isn't it getting time to think to the best ways to obtain a mutation of all health professions confronted to the new era of the "information society" by emphasizing the human approach through a new education?[4].

\section{References}

1. FH Roger (1981) The Minimum Basic Data Set for Hospital Statistics in the EEC, CEC, Luxembourg, p. 151.

2. Robert B Fetter, Youngsoo Shin, Jean L Freeman, Richard F Averill, John D Thompson (1980) Case-mix definition by Diagnosis Related Groups, Medical Care 18(supp 2): 1-52.

3. Lawrence D (1971) WEED Medical Records, Medical Education and Patient Care, the Press of Case Western Reserve University, p. 292.

4. (2018) Francis Roger France Trois clés pour l'Europe du 21ème siècle (including more than 200 references), (Editions Européennes Universitaires (EUE), p. 282.

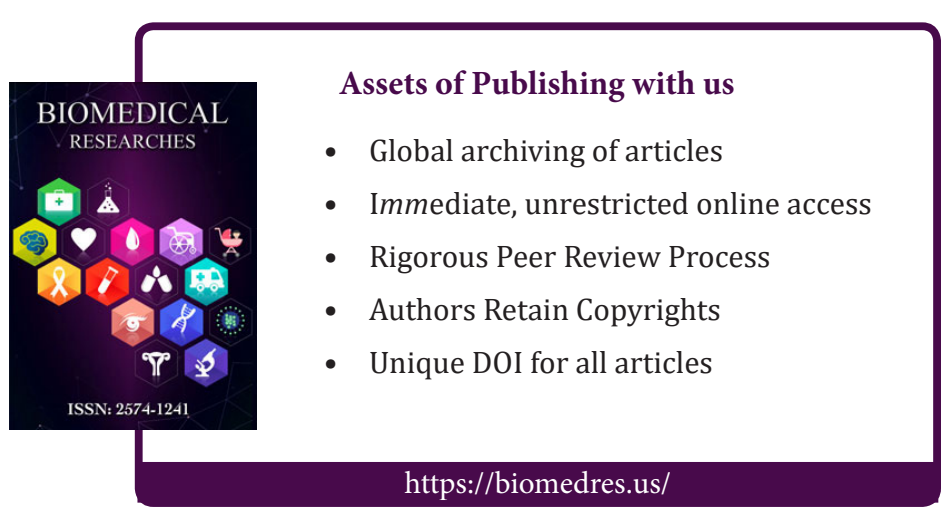

